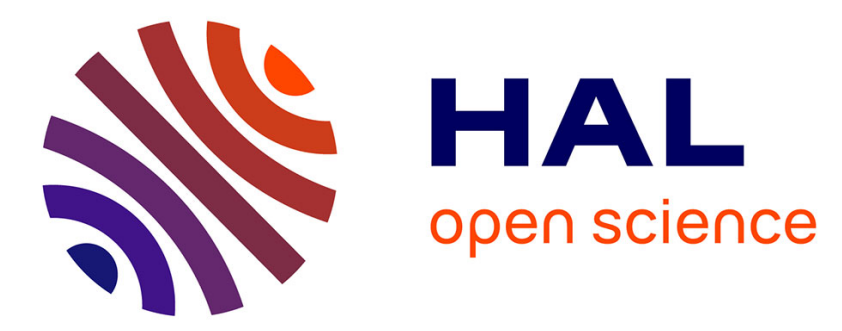

\title{
Extended spectrum of antibiotic susceptibility for tuberculosis, Djibouti
}

Feriel Bouzid, Helene Astier, Djaltou Aboubaker Osman, Emilie Javelle, Mohamed Osman Hassan, Fabrice Simon, Eric Garnotel, Michel Drancourt

\section{- To cite this version:}

Feriel Bouzid, Helene Astier, Djaltou Aboubaker Osman, Emilie Javelle, Mohamed Osman Hassan, et al.. Extended spectrum of antibiotic susceptibility for tuberculosis, Djibouti. International Journal of Antimicrobial Agents, 2018, 51 (2), pp.235-238. 10.1016/j.ijantimicag.2017.07.007 . hal-01780646

\section{HAL Id: hal-01780646 \\ https://hal.science/hal-01780646}

Submitted on 11 Apr 2019

HAL is a multi-disciplinary open access archive for the deposit and dissemination of scientific research documents, whether they are published or not. The documents may come from teaching and research institutions in France or abroad, or from public or private research centers.
L'archive ouverte pluridisciplinaire HAL, est destinée au dépôt et à la diffusion de documents scientifiques de niveau recherche, publiés ou non, émanant des établissements d'enseignement et de recherche français ou étrangers, des laboratoires publics ou privés. 
Short Communication

\title{
Extended spectrum of antibiotic susceptibility for tuberculosis, Djibouti
}

\author{
Fériel Bouzid ${ }^{\mathrm{a}, \mathrm{b}}$, Hélène Astier ${ }^{\mathrm{c}}$, Djaltou Aboubaker Osman ${ }^{\mathrm{d}}$, Emilie Javelle ${ }^{\mathrm{c}}$, \\ Mohamed Osman Hassan ${ }^{\mathrm{e}}$, Fabrice Simon ${ }^{\mathrm{f}}$, Eric Garnotel ${ }^{\mathrm{c}}$, Michel Drancourt ${ }^{\mathrm{a}, *}$ \\ a Aix Marseille Université, URMITE, UMR CNRS 7278, IRD 198, INSERM 1095, IHU Méditerranée Infection, Marseille 13005, France \\ b Aix-Marseille Univ, CNRS, EIPL, Marseille, France \\ ' Hôpital d'instruction des armées Alphonse Laveran, Marseille, France \\ d Centre d'Études et de Recherche de Djibouti (CERD), Institut de Recherche Médicinale (IRM), Djibouti, Djibouti \\ e Hôpital pneumo-phtisiologie "Chakib Saad Omar», Djibouti \\ fôle Formation-Recherche, Hôpital d'instruction des armées Alphonse Laveran, Marseille, France
}

\section{A R T I C L E I N F O}

\section{Article history:}

Received 28 March 2017

Accepted 8 July 2017

Editor: B. Gold

\section{Keywords:}

Mycobacterium tuberculosis

Mycobacterium canettii

'Mycobacterium simulans'

Mycobacterium kansasii

Horn of Africa

\begin{abstract}
A B S T R A C T
In the Horn of Africa, there is a high prevalence of tuberculosis that is reported to be partly driven by multidrug-resistant (MDR) Mycobacterium tuberculosis strictu sensu strains. We conducted a prospective study to investigate $M$. tuberculosis complex species causing tuberculosis in Djibouti, and their in vitro susceptibility to standard anti-tuberculous antibiotics in addition to clofazimine, minocycline, chloramphenicol and sulfadiazine. Among the 118 mycobacteria isolates from 118 successive patients with suspected pulmonary tuberculosis, 111 strains of $M$. tuberculosis, five Mycobacterium canettii, one 'Mycobacterium simulans' and one Mycobacterium kansasii were identified. Drug-susceptibility tests performed on the first 78 isolates yielded nine MDR M. tuberculosis isolates. All isolates were fully susceptible to clofazimine, minocycline and chloramphenicol, and 75 of 78 isolates were susceptible to sulfadiazine. In the Horn of Africa, patients with confirmed pulmonary tuberculosis caused by an in vitro susceptible strain may benefit from anti-leprosy drugs, sulfamides and phenicol antibiotics.
\end{abstract}

(c) 2017 Elsevier B.V. and International Society of Chemotherapy. All rights reserved.

\section{Introduction}

Tuberculosis remains a major deadly infectious disease worldwide, with 10.4 million new cases and 1.4 million deaths from the disease in 2015 [1]. However, the burden of tuberculosis varies greatly from one country to another, as illustrated by the ten-fold variation in incidence from $27 / 100000$ inhabitants in wealthy regions of America to an average rate of 275/100 000 inhabitants in Africa [1]. The Horn of Africa, comprising Djibouti, Ethiopia, Somalia and Eritrea, is a hotspot for tuberculosis, with a high incidence of 378/ 100000 inhabitants in Djibouti and 192/100 000 inhabitants in Ethiopia in 2015 [1]. In Djibouti, the high prevalence of tuberculosis has been reported to be partly driven by so-called multidrugresistant (MDR) Mycobacterium tuberculosis isolates [2,3], defined as being resistant to at least isoniazid and rifampicin [4]. Moreover, in Djibouti, tuberculosis is caused not only by $M$. tuberculosis isolates, but also by Mycobacterium canettii in 3\% and up to $6 \%$ of cases [3,5]. Notably, M. canettii organisms are naturally resistant to pyrazinamide and streptomycin [6].

\footnotetext{
* Corresponding author. URMITE, UMR CNRS 7278, IRD 198, INSERM U1095, IHU Méditerranée Infection, 19-21 Bd Jean Moulin, 13005 Marseille, France.

E-mail address: michel.drancourt@univ-amu.fr (M. Drancourt).
}

With a view to updating information on the microbiology of pulmonary tuberculosis in Djibouti, we prospectively identified mycobacterial isolates from patients presenting with clinicallysuspected pulmonary tuberculosis, and extended in vitro drug susceptibility tests for classic anti-tuberculous antibiotics to include clofazimine [7], minocycline [8], chloramphenicol [9] and sulfadiazine [10], all of which were shown to inhibit mycobacteria.

\section{Materials and methods}

\subsection{Patients and sample processing}

This study was approved by the IHU Méditerranée Infection, Ethics Committee Approval ${ }^{\circ}$ 2016-025, Marseille, France. The study was conducted in collaboration with the Hôpital Pneumo-phtisiologie Chakib Saad Omar in Djibouti as part of their routine activity. All patients with suspected pulmonary tuberculosis who attended this hospital between May and December 2016 were included in the study. Informed and consenting patients answered an anonymized questionnaire containing personal information (age, sex, nationality, address, job and travel out of Djibouti), human immunodeficiency virus (HIV) status, medical history and reported tuberculosis cases in their family. Collected sputa were systematically examined by microscopy observation after Ziehl-Neelsen staining. Samples were 
stored at $4{ }^{\circ} \mathrm{C}$ before shipment. Every two weeks, samples that tested positive for microscopic detection of acid-fast bacilli were shipped to the biology laboratory in the Hôpital d'Instruction des Armées Alphonse Laveran, Marseille, France, with an average transport time of eight days. Samples were then decontaminated using the BD BBL ${ }^{\mathrm{TM}}$ MycoPrep ${ }^{\mathrm{TM}}$ Mycobacterial System Digestion/Decontamination Kit (Becton Dickinson, Le Pont-de-Claix, France), and cultured on Coletsos medium (Bio-Rad, Marnes-la-Coquette, France) and on egg-based Lowenstein-Jensen medium (Bio-Rad, Marnes-la-Coquette, France), at $37^{\circ} \mathrm{C}$ for 60 days. Colonies of positive cultures were stained with fluorescent auramine-O staining (Sigma, Saint-Quentin Fallavier, France). The presence of mycobacteria was confirmed by detecting stained bacilli after fluorescence microscopy examination.

\subsection{Identification tests}

Mycobacteria were identified by matrix-assisted laser desorption ionization time-of-flight mass spectrometry (MALDI-TOFMS) after direct deposit of the colonies [11]. Briefly, colonies were spotted on a MALDI-TOF target plate and then covered with $1 \mu \mathrm{L}$ matrix solution. After drying, the target plate was introduced into the Microflex LT MALDI-TOF mass spectrometer (Bruker Daltonics, Germany) for analysis. Generated spectra were compared with the Bruker peptide profile database and identification was performed using the pattern-matching process as previously described [11]. 16S rRNA and $r p o B$ gene sequencing [12,13] were then performed to identify non-tuberculous mycobacteria. $M$. tuberculosis complex isolates were identified at the species level by specific multiplex polymerase chain reaction (PCR) amplification of regions of difference (RD) RD4, RD9 and RD12 [14] using M. tuberculosis H37Rv, Mycobacterium bovis BCG Pasteur and M. canettii CIP $140010059^{\mathrm{T}}$ as positive controls and Mycobacterium smegmatis $\mathrm{mc}^{2}$ 155 as a negative control. $M$. canettii identification was confirmed by partial hsp65 gene sequencing [6] and quantitative real time PCR (qPCR) targeting the cobF gene using cobF_Forward_5'-GCGACTGCTCGTTTCAGG-3', cobF_Reverse_5'-GATGCGTGTCCCGACCTC-3' and cobF_Probe_5'6FAM- CCGGACACCCGAATCTGGTGG-3'. For all tuberculous isolates, real-time PCR targeting $M$. tuberculosis-specific deletion 1 (TbD1) was performed using TbD1_ Forward 5'-CAAA GGAACCGCGAAAGTTA-3', TbD1_ Reverse 5'-ACCGTGATAA GCACCAGGAC-3' and TbD1_Probe_5'-6FAM- TCGCGGTGATGTT GCTCTTCG-3'. All qPCR experiments were conducted using the CFX96 ${ }^{\circledR}$ qPCR, incorporating qPCR reagents (Takyon, Eurogentec, Liège, Belgium). The qPCR cycle was $50{ }^{\circ} \mathrm{C}$ for two minutes, $95^{\circ} \mathrm{C}$ for five minutes followed by 40 cycles of $95^{\circ} \mathrm{C}$ for one second and $60{ }^{\circ} \mathrm{C}$ for 35 seconds and finally $45^{\circ} \mathrm{C}$ for 30 seconds.

\subsection{Drug susceptibility testing}

Drug susceptibility tests for streptomycin, isoniazid, rifampicin, ethambutol and pyrazinamide were performed in liquid medium by an automated BACTEC ${ }^{\text {TM }}$ MGIT ${ }^{\mathrm{TM}} 960$ method using the BACTEC ${ }^{\text {TM }}$ MGITTM 960 SIRE kit and the BACTEC $^{\text {TM }}$ MGITTM $^{\text {TM }} 90$ PZA kit (Becton Dickinson). Final concentrations in MGIT tubes were $1 \mu \mathrm{g} / \mathrm{mL}$ for streptomycin, $0.10 \mu \mathrm{g} / \mathrm{mL}$ for isoniazid, $1 \mu \mathrm{g} / \mathrm{mL}$ for rifampicin, $5 \mu \mathrm{g} / \mathrm{mL}$ for ethambutol and $100 \mu \mathrm{g} / \mathrm{mL}$ pyrazinamide according to the manufacturer's recommendations (Becton Dickinson). $M$. tuberculosis H37Rv was used as a control strain. Further susceptibility tests were performed by molecular hybridization using the GenoType MTBDRplus kit (Hain LifeScience) to detect rifampicin and isoniazid resistance. For the clofazimine, minocycline, chloramphenicol and sulfadiazine (CMCS) susceptibility test, mycobacterial liquid cultures were calibrated at a final concentration of $10^{5}$ colonyforming units/mL according to McFarland standards. Drug stock solutions in dimethyl sulfoxide (Euromedex, Souffelweyersheim, France) were stored at $-20^{\circ} \mathrm{C}$, except for sulfadiazine, which was freshly prepared. The applied drug concentrations were $1.5 \mu \mathrm{g} / \mathrm{mL}$ for clofazimine, $4 \mu \mathrm{g} / \mathrm{mL}$ for minocyline, $5 \mu \mathrm{g} / \mathrm{mL}$ for chloramphenicol and $20 \mu \mathrm{g} / \mathrm{mL}$ for sulfadiazine, based on previous studies [7-10]. The CMCS susceptibility test was performed by adapting the automated MGIT960 method. For each isolate tested, one drug-free growth control tube and four additional drug-containing tubes were inoculated with $0.5 \mathrm{~mL}$ mycobacterial suspension. An antimicrobial susceptibility testing set comprising the five tubes was incubated in the Bactec MGIT 960 instrument (Becton Dickinson). Results were analysed by the Bactec MGIT 960 system within seven to ten days and interpreted as previously described [15].

\section{Results}

Over an eight-month prospective study, 200 samples and questionnaires from 200 patients were sent to the laboratory. Seventy samples did not grow and 12 were contaminated. Of the 118 mycobacteria isolated from 118 sputa, MALDI-TOF-MS identified $116 M$. tuberculosis complex isolates and one M. kansasii isolate, and there was one unidentified isolate. Of $116 \mathrm{M}$. tuberculosis complex isolates, the analysis of restriction digests (RDs) by PCR multiplex yielded $111 \mathrm{M}$. tuberculosis and five $\mathrm{M}$. canettii isolates. Molecular characterization showed that 51/111 (46\%) M. tuberculosis isolates were TbD1-positive. The five M. canettii isolates were firmly identified by cobF gene-positivity, the presence of TbD1 region and a C-to-T transition in the hsp65 gene. One ' $M$. simulans' and one $M$. kansasii isolates were firmly identified based on a $16 \mathrm{~S}$ rRNA and rpoB gene sequences with $99 \%$ sequence similarity with the references. For the $110 \mathrm{M}$. tuberculosis-infected patients whose sex was known, 79 (71.8\%) were men and 31 (28.1\%) were women, and the average age was 33 (range 13-73) years. Of the 108 patients whose geographical origin was known, 92 patients $(85 \%)$ were Djiboutian, 13 (12\%) were Ethiopian, two were Somalian and one was Yemenite. Only eight patients (7.2\%) reported a previous history of treatment for tuberculosis. Information on HIV status was known for 88 of the M. tuberculosis-infected patients, of whom three (3.4\%) were HIV-positive. The five patients infected by $M$. canettii were all Djiboutian (three men and two women, average age: 34 years) who originated from five different neighborhoods (Table 1). These patients reported no tuberculosis history and were HIV-negative. Three of these patients reported a stay in Somaliland and one reported a stay in Ethiopia (Table 1). Travel to Somaliland in $3 / 5(60 \%)$ of $M$. canettii-infected patients was

Table 1

Synopsis of five patients diagnosed with M. canettii pulmonary tuberculosis, Djibouti.

\begin{tabular}{|c|c|c|c|c|c|c|c|}
\hline Isolate & Sex & Age & Nationality & Job & Address in Djibouti & Travel outside Djibouti & HIV status \\
\hline DJ 480 & M & 30 & DJ & No occupation & Quartier 3 & Somaliland 2015 & No \\
\hline DJ 514 & $\mathrm{~F}$ & 41 & DJ & No occupation & Quartier 7 bis & Somaliland 2014-2015 & No \\
\hline DJ 517 & M & 27 & DJ & No occupation & Cité Poudrière & No & No \\
\hline DJ 613 & $\mathrm{~F}$ & 27 & DJ & No occupation & Quartier 2 & Somaliland 2012 & No \\
\hline DJ 734 & M & 47 & DJ & Cooking & Quartier 7 & Ethiopia 2015 & No \\
\hline
\end{tabular}

M: men, F: women, DJ: Djiboutian, HIV: human immunodeficiency virus. 
Table 2

In vitro drug susceptibility testing of mycobacteria isolated from patients with suspected pulmonary tuberculosis, Djibouti.

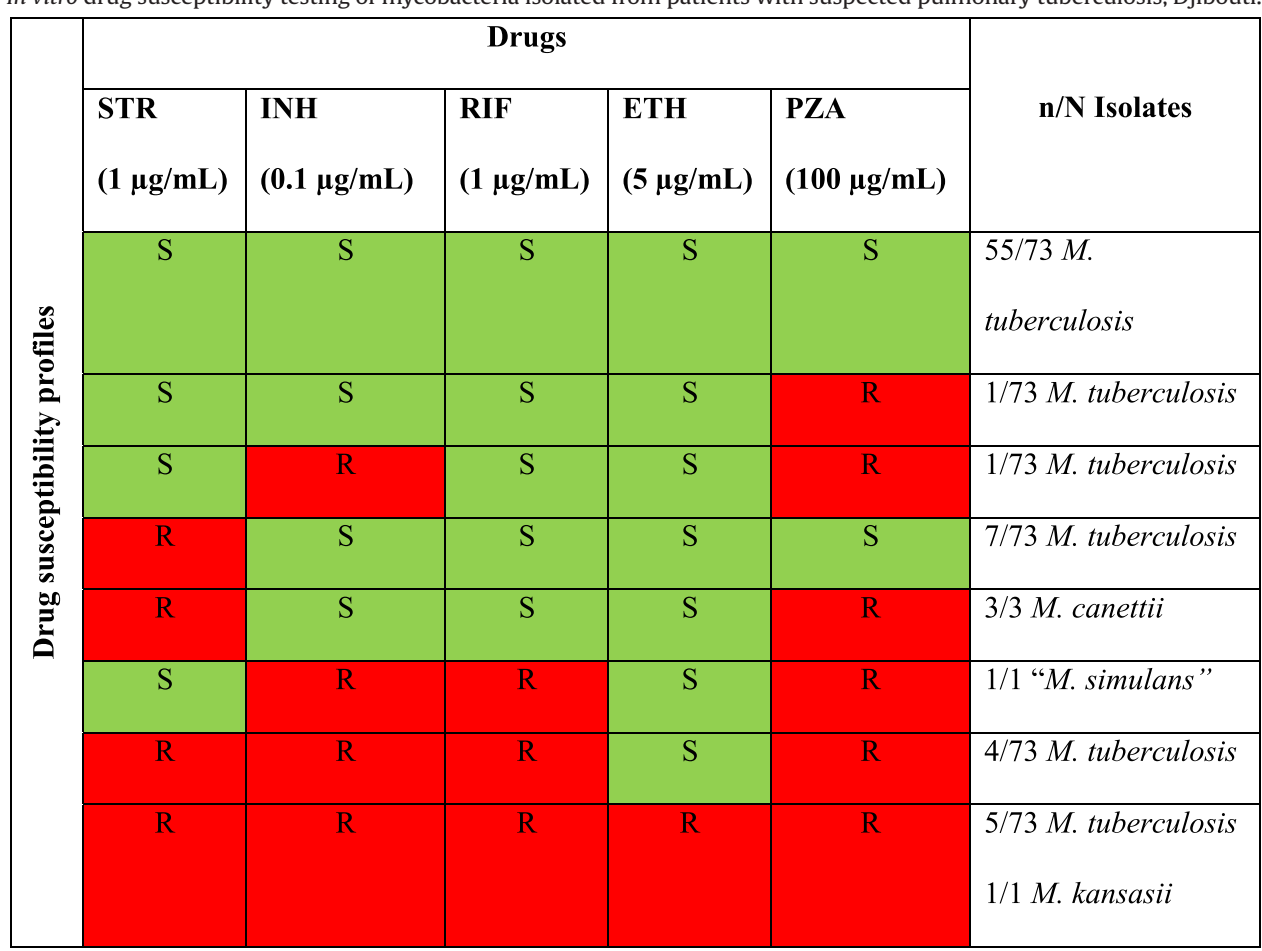

STR: streptomycin, INH: isoniazid, RIF: rifampicin, ETH: ethambutol, PZA: pyrazinamide, green square, S: susceptible; red square, $\mathrm{R}$ : resistant, $\mathrm{n} / \mathrm{N}$ : number of obtained profiles/total number of isolates.

significantly higher than the $4 / 111$ (3.6\%) of $M$. tuberculosisinfected patients $(P=0.001$, Fisher's exact test), but this was not true for travel to Ethiopia ( $P=1$, Fisher's exact test). One ' $M$. simulans' isolate with uncertain pathogenicity was documented in a 40 -yearold Djiboutian man living in Balbala, a suburb of Djibouti-town. This HIV-negative patient had been initially diagnosed with cavitary pulmonary disease and had been unsuccessfully treated for non-documented MDR tuberculosis. One M. kansasii-infected patient was a 46-year-old Djiboutian man working as a painter and living in Quartier 7 of Djibouti. He reported a stay of one year in Somaliland in 2012. The patient reported no tuberculosis history, was HIV-negative and had been previously diagnosed with nondocumented MDR tuberculosis.

Drug susceptibility testing was performed for 78/118 (66\%) isolates including $73 \mathrm{M}$. tuberculosis, three $M$. canettii, one 'M. simulans' and one M. kansasii isolates. As for SIRE and PZA susceptibility tests, 55/78 (70\%) isolates were susceptible to all anti-tuberculosis drugs tested (Table 2). The three $M$. canettii isolates were resistant to streptomycin and pyrazinamide. The ' $M$. simulans' isolate was resistant to rifampicin, isoniazid and pyrazinamide. The M. kansasii isolate was resistant to the standard concentrations applied for the anti-tuberculous antibiotics. Nine MDR among the $73 \mathrm{M}$. tuberculosis isolates were obtained by the BACTEC method and confirmed by the molecular hybridization technique. HIV status was reported for seven MDR-tuberculosis patients, all of whom were HIV-negative. Past medical history of tuberculosis was indicated in four of the nine cases of MDR M. tuberculosis. For the CMCS susceptibility test, all 78 tested mycobacteria, including the nine MDR M. tuberculosis isolates, were inhibited by $1.5 \mu \mathrm{g} / \mathrm{mL}$ clofazimine, $4 \mu \mathrm{g} / \mathrm{mL}$ minocycline, $5 \mu \mathrm{g} / \mathrm{mL}$ chloramphenicol and $20 \mu \mathrm{g} / \mathrm{mL}$ sulfadiazine, except for three $M$. tuberculosis isolates, including two MDR that were resistant to sulfadiazine.

\section{Discussion}

In Djibouti, pulmonary tuberculosis is routinely diagnosed based on clinical data and positive microscopic observation of a sputum smear following Ziehl-Neelsen staining [3]. These criteria do not provide information on the mycobacterial species causing the tuberculosis and leave scope for the possibility that some patients who are clinically diagnosed with pulmonary tuberculosis are, in fact, infected by other mycobacteria. Accordingly, our prospective study revealed $M$. canettii in some patients in Djibouti, in line with observations previously reported in Djibouti $[3,5]$. In addition, we recovered one strain of $M$. kansasii and one strain of ' $M$. simulans', which had not previously been reported in Djibouti. However, the interpretation of these two isolates remains uncertain given the absence of repeated sampling from these patients, and co-infection with tuberculous mycobacteria cannot be excluded for the same reason [16]. Indeed, it is the second isolate of ' $M$. simulans' infection worldwide and, notably, the index case reported a few years ago was a Somalian patient diagnosed with cavitary pulmonary disease mimicking MDR tuberculosis [17]. The patient reported in this paper had a similar medical history, yet denied having had any contact with Somalia. A few studies identified non-tuberculous mycobacteria as being responsible for pulmonary infection in Djibouti, including Mycobacterium chelonae, Mycobacterium fortuitum and Mycobacterium peregrinum [3].

Our in vitro susceptibility data yielded a $9 / 73$ (12.3\%) frequency of MDR among $M$. tuberculosis isolates, which is significantly lower than $72 \%(23 / 32)\left(P=1.10^{-9}, \chi^{2}\right.$ test $)$ and $57 \%(51 / 88)\left(P=2.10^{-9}, \chi^{2}\right.$ test) previously reported in Djibouti $[2,3]$. The fact that previous retrospective studies incorporated data from 2007-2011 [2,3], and that tested samples had been collected from hospitalized patients, may account for differences in estimations of the actual MDR rate [2]. Moreover, because the present strain series is limited in terms of 
the number of strains and the length of the collection, it may not be representative of the overall current epidemiology of tuberculosis in Djibouti. Only larger and longer-term studies will give a reasonable overview of the situation. We observed that one 'M. simulans' isolate was resistant to isoniazid and rifampicin, as previously reported [17], and was susceptible to streptomycin and resistant to pyrazinamide, contrary to that reported for the first strain [17]. Whether this observation indicates a natural heterogeneity of antibiotic susceptibility pattern in 'M. simulans' will depend on the observation of further isolates. M. kansasii is naturally resistant to pyrazinamide [18], but higher inhibitory concentrations of streptomycin, isoniazid, rifampicin and ethambutol than those applied in our study are required [19].

In the absence of any standardized published protocol to test the in vitro susceptibility of $M$. tuberculosis complex mycobacteria to the anti-leprosy antibiotics, sulfadiazine and chloramphenicol, we tested a single concentration in the upper limit of the inhibitory concentrations previously reported [7-10]. Bearing this limit in mind, we observed that all M. tuberculosis complex isolates, including nine MDR isolates, were susceptible to clofazimine, minocyline and chloramphenicol, and 94\% were susceptible to sulfadiazine. In this paper, we report the antimicrobial activity of clofazimine, minocyline, chloramphenicol and sulfadiazine against ' $M$. simulans' and $M$. canettii, thereby confirming previous observations $[10,17]$. Moreover, the inhibition of M. kansasii by chloramphenicol is herein demonstrated for the first time.

\section{Conclusions}

This study illustrates the impact of accurate identification of mycobacteria recovered from respiratory tract specimens to confirm and refine the diagnosis of tuberculosis in countries with a high incidence of tuberculosis, where several M. tuberculosis complex species are circulating. In Djibouti, mycobacteria responsible for suspected pulmonary tuberculosis were susceptible in vitro to at least three different antibiotics from an extended spectrum of antituberculous, anti-leprosy, sulfamide and phenicol antibiotics. We propose that such strains could be qualified as 'multidrugsusceptible', opposing the definition of multidrug-resistant strains.

Anti-leprosy, sulfamide and phenicol antibiotics are all orally administered, may be included in Directly Observed Treatment Short course strategy, and their potential toxicity is well established, given the 30-50 years of prescription worldwide [20]. Data reported here indicate that these antibiotics could be considered during the empirical treatment of patients presenting with confirmed tuberculosis in the Horn of Africa.

Funding: This study was financially supported by URMITE, IHU Méditerranée Infection, Marseille, France.

Competing interests: None declared.

Ethical approval: This study was approved by the IHU Méditerranée Infection, Ethics Committee Approval n²016-025, Marseille, France.

\section{References}

[1] World Health Organization (WHO). Global tuberculosis report. 2016. Available from: http://apps.who.int/iris/bitstream/10665/250441/1/9789241565394 -eng.pdf?ua $=1 ; 2016$. [Accessed 3 October 2017].

[2] Millan-Lou MI, Olle-Goig JE, Tortola MT, Martin C, Samper S. Mycobacterial diversity causing multi- and extensively drug-resistant tuberculosis in Djibouti, Horn of Africa. Int J Tuberc Lung Dis 2016;20:150-3.

[3] Boyer-Cazajous G, Martinaud C, Dehan C, Hassan MO, Gaas Y, Chenilleau-Vidal MC, et al. High prevalence of multidrug resistant tuberculosis in Djibouti: a retrospective study. J Infect Dev Ctries 2014;8:233-6.

[4] Centers for Disease Control and Prevention (CDC). Emergence of Mycobacterium tuberculosis with extensive resistance to second-line drugs-worldwide, 2000-2004. MMWR Morb Mortal Wkly Rep 2006;55:301-5.

[5] Koeck JL, Bernatas JJ, Gerome P, Fabre M, Houmed A, Herve V, et al. [Epidemiology of resistance to antituberculosis drugs in Mycobacterium tuberculosis complex strains isolated from adenopathies in Djibouti. Prospective study carried out in 1999]. Med Trop (Mars) 2002;62:70-2.

[6] Goh KS, Legrand E, Sola C, Rastogi N. Rapid differentiation of 'Mycobacterium canettii' from other Mycobacterium tuberculosis complex organisms by PCR-restriction analysis of the hsp65 gene. J Clin Microbiol 2001;39:3705-8.

[7] Jagannath C, Reddy MV, Kailasam S, O'Sullivan JF, Gangadharam PR. Chemotherapeutic activity of clofazimine and its analogues against Mycobacterium tuberculosis. In vitro, intracellular, and in vivo studies. Am J Respir Crit Care Med 1995;151:1083-6.

[8] Wallace RJ Jr, Brown-Elliott BA, Crist CJ, Mann L, Wilson RW Comparison of the in vitro activity of the glycylcycline tigecycline (formerly GAR-936) with those of tetracycline, minocycline, and doxycycline against isolates of nontuberculous mycobacteria. Antimicrob Agents Chemother 2002;46:3164-7.

[9] Sohaskey CD. Enzymatic inactivation and reactivation of chloramphenicol by Mycobacterium tuberculosis and Mycobacterium bovis. FEMS Microbiol Lett 2004;240:187-92.

[10] Ameen SM, Drancourt M. In vitro susceptibility of Mycobacterium tuberculosis to trimethoprim and sulfonamides in France. Antimicrob Agents Chemother 2013;57:6370-1.

[11] Zingue D, Flaudrops C, Drancourt M. Direct matrix-assisted laser desorption ionisation time-of-flight mass spectrometry identification of mycobacteria from colonies. Eur J Clin Microbiol Infect Dis 2016;35:1983-7.

[12] Adekambi T, Berger P, Raoult D, Drancourt M. rpoB gene sequence-based characterization of emerging non-tuberculous mycobacteria with descriptions of Mycobacterium bolletii sp. nov., Mycobacterium phocaicum sp. nov. and Mycobacterium aubagnense sp. nov. Int J Syst Evol Microbiol 2006;56:133-43.

[13] Drancourt M, Bollet C, Carlioz A, Martelin R, Gayral JP, Raoult D. 16S ribosomal DNA sequence analysis of a large collection of environmental and clinical unidentifiable bacterial isolates. J Clin Microbiol 2000;38:3623-30.

[14] Warren RM, Gey van Pittius NC, Barnard M, Hesseling A, Engelke E, de Kock $\mathrm{M}$, et al. Differentiation of Mycobacterium tuberculosis complex by PCR amplification of genomic regions of difference. Int J Tuberc Lung Dis 2006;10:818-22.

[15] Springer B, Lucke K, Calligaris-Maibach R, Ritter C, Bottger EC. Quantitative drug susceptibility testing of Mycobacterium tuberculosis by use of MGIT 960 and EpiCenter instrumentation. J Clin Microbiol 2009;47:1773-80.

[16] Griffith DE, Aksamit T, Brown-Elliott BA, Catanzaro A, Daley C, Gordin F, et al. An official ATS/IDSA statement: diagnosis, treatment, and prevention of nontuberculous mycobacterial diseases. Am J Respir Crit Care Med 2007; 175:367-416.

[17] Tortoli E, Rogasi PG, Fantoni E, Beltrami C, De Francisci A, Mariottini A. Infection due to a novel mycobacterium, mimicking multidrug-resistant Mycobacterium tuberculosis. Clin Microbiol Infect 2010;16:1130-4.

[18] Sun Z, Zhang Y. Reduced pyrazinamidase activity and the natural resistance of Mycobacterium kansasii to the antituberculosis drug pyrazinamide. Antimicrob Agents Chemother 1999;43:537-42.

[19] da Silva Telles MA, Chimara E, Ferrazoli L, Riley LW. Mycobacterium kansasii: antibiotic susceptibility and PCR-restriction analysis of clinical isolates. J Med Microbiol 2005;54:975-9.

[20] Brouqui P, Quenard F, Drancourt M. Old antibiotics for emerging multidrugresistant/extensively drug-resistant tuberculosis (MDR/XDR-TB). Int J Antimicrob Agents 2017;49:554-7. 